\title{
Représentations, attitudes, stylisation vocale des accents en formation Français Langue d'Insertion/ d'Intégration
}

Representations, attitudes and vocal stylisation associated with accents in compulsory French language courses for immigrants

\section{Myriam Dupouy}

\section{OpenEdition \\ Journals}

Édition électronique

URL : https://journals.openedition.org/lbl/290

DOI : $10.4000 / \mathrm{lbl} .290$

ISSN : 2727-9383

Éditeur

Université de Bretagne Occidentale - UBO

\section{Édition imprimée}

Date de publication : 1 mai 2017

Pagination : 195-207

ISBN : 979-10-92331-31-8

ISSN : $1270-2412$

\section{Référence électronique}

Myriam Dupouy, «Représentations, attitudes, stylisation vocale des accents en formation Français Langue d'Insertion/d'Intégration », La Bretagne Linguistique [En ligne], 21 | 2017, mis en ligne le 01 mai 2020, consulté le 27 mars 2022. URL : http://journals.openedition.org//bl/290 ; DOI : https://doi.org/ $10.4000 / / b l .290$

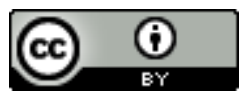

La Bretagne Linguistique est mise à disposition selon les termes de la Licence Creative Commons Attribution 4.0 International. 


\title{
Représentations, attitudes, stylisation vocale des accents en formation Français Langue d'Insertion/d'Intégration
}

\begin{abstract}
$\mathrm{M}$ a recherche doctorale ${ }^{1}$ en sociolinguistique porte sur les représentations et attitudes relatives aux accents et à la musicalité de la langue française en formation FLI, Français Langue d'Insertion/Intégration pour adultes en situation de migration en région Bretagne. La problématique est la suivante : en quoi la prise en compte des représentations et attitudes liées aux accents de la langue française représente-t-elle un enjeu et a-t-elle une incidence sur le déroulement de la formation en FLI, tant du point de vue de l'apprenant que de celui du formateur? Cette recherche porte aussi bien sur la mise en lumière du fonctionnement des phénomènes questionnés que sur leurs conséquences sur l'objectif de la formation cible, à savoir l'appropriation du français par des adultes migrants dans une visée d'insertion ${ }^{2}$.
\end{abstract}

* Doctorante en sociolinguistique au CRBC (EA 4451), sous la direction de Ronan Calvez et d'Aude Bretegnier (CREN - EA 2661).

1. Cette recherche doctorale a été financée par le ministère de l'Enseignement supérieur et de la Recherche pour une durée de trois ans.

2. Aude BretegnieR, «Interroger le sens et les enjeux des projets d'appropriation du français langue d'insertion : ouverture d'un champ de recherches interventions ", Glottopol, n 11, 2008 ; Aude BrETEGNIER (dir.), Formation linguistique en contextes d'insertion. Compétences professionnelles, posture, professionnalité : concevoir un cadre de référence(s), Collection «Transversales », Berne, Peter Lang, 2011. 
Cette contribution traitera d'un aspect méthodologique connu des sociolinguistes, et plus largement des chercheurs en sciences sociales, puisqu'il s'agit de la question des transcriptions d'entretiens et des interactions saisies ${ }^{3}$ en contexte particulier (dans notre cas, en contexte-classe). Ce point, bien que largement traité dans la littérature, reste néanmoins spécifique à chaque objet de recherche et résulte de choix réalisés par le chercheur en amont et en cours de recherche, en interaction avec les premières données recueillies. Dans notre cas, ce moment charnière de la transcription, bien qu'ayant été anticipé et questionné lors de la mise en place du protocole d'enquête, s'est avéré fructueux au niveau d'une première analyse de contenu, et ce grâce à l'adaptation du concept de stylisation que je développerai après avoir présenté le contexte de la recherche.

\section{Mise en contexte de la recherche doctorale}

\section{Accents - Musicalité}

Les accents sont présents au quotidien, au gré des rencontres et des interactions ou dans les médias. Les accents font en effet l'objet de nombreux commentaires, qu'il s'agisse de leur réception ou de leur production. Mais qu'entend-on par accents ? Les définitions semblent aussi diverses que les accents eux-mêmes, tant c'est un terme ancré dans le lexique commun. Là où les linguistes le définissent par des caractéristiques identifiables et mesurables et donc comme un élément de la phonologie propre à chaque langue, il semble relever davantage de la production. Il en est de même en didactique des langues où aborder l'accent revient, en grande partie, à enseigner des règles permettant d'expliciter ce qui compose (entre autres éléments supra-segmentaux) le rythme de la langue cible.

Cependant, l'accent, ou plutôt, les accents dépassent ce cadre strictement linguistique (au sens classique du terme) puisqu'ils font l'objet de représentations, tant du point de vue de la production que de la réception. L'objet de recherche « accents ", peu étudié jusqu'alors ${ }^{4}$,

3. Le verbe utilisé ici rend compte de l'impossibilité de capter (audio ou vidéo) les interactions en contexte-classe au sein des lieux d'enquête.

4. Noter l'ouvrage précurseur de Rosina LIPPI-GREEN, English with an accent : Language, Ideology and Discrimination in the United States, London, Routledge, 2012 [1997]. 
interroge les sociolinguistes et anthropologues du langage, ce qui élargit le spectre de sa définition et ne le confine plus au cadre d'études strictement linguistiques.

«Non seulement les accents ne sont pas réductibles, pour les locuteurs, à des prononciations (car leur conception de l'accent déborde ce niveau de description), mais en outre l'accent est inscrit dans une relation d'altérité et de pouvoir, prenant ainsi une dimension éthique et politiquement incontournable ${ }^{5}$.»

Cette notion complexe et plurielle d'accent, se ressent donc d'un point de vue phonologique mais aussi et surtout sous un angle sociolinguistique. C'est donc par le prisme de nos représentations, nos constructions socioculturelles, et pédagogiques si nous sommes enseignants, que l'on peut assez facilement penser identifier un accent marseillais, breton, québécois, mais aussi de Neuilly ou dit des banlieues par exemple.

(...) la perception de l'accent est un préalable à la catégorisation des locuteurs qui repose largement sur lui. La catégorisation et les évaluations qu'elle implique se font sur la base d'indicateurs socioperceptifs et d'évaluateurs socioculturels ${ }^{6}$.

Il ne s'agit plus ici de phonologie, puisque cela n'altère normalement pas la communication, mais bel et bien de variations qui ont une influence sur la chaîne sonore : variations géographiques ou régionales, mais aussi variations sociales (diastratiques).

Il est donc possible, à partir de l'élargissement de leur définition, d'étudier les accents en tant que composante des discriminations langagières par exemple ${ }^{7}$. La question ici touche l'enseignement et l'apprentissage, il est donc nécessaire d'ajouter à cette définition une dimension

5. Médéric GASQUET-CYRUS, «L'accent: concept (socio)linguistique ou catégorie de sens commun? ", dans Pour une épistémologie de la sociolinguistique, Limoges, LambertLucas Éditions, 2010, p. 179-189.

6. Christine DEPREZ, «Remarques socio-didactiques sur l'accent en français langue étrangère ", dans Année européenne du dialogue interculturel : communiquer avec les languescultures, Actes du colloque international 2008, Université Aristote de Thessaloniki, 2008, p. 151-161.

7. Voir à ce sujet le récent ouvrage de Philippe BLANCHET, Discriminations : combattre la glottophobie, Paris, Éditions Textuel, 2016. 
didactique. En effet, si chacun perçoit des accents, qu'ils soient géographiques, sociaux, valorisés ou non, incompréhensibles ou non, ces perceptions semblent décuplées lorsque le contexte présente de facto un contact de langues, comme c'est le cas en formation FLI. Dans ce cadre spécifique, multilingue, les réactions, les sentiments et les jugements de valeurs sont donc récurrents.

Ce sont ces attitudes et représentations que nous cherchons à comprendre et à analyser dans une perspective interactionniste.

\section{Le FLI et le public}

La recherche est menée auprès des stagiaires adultes en situation de migration qui suivent une formation linguistique en Français Langue d'intégration. Le FLI, qui vise « un usage quotidien de la langue française et l'apprentissage des outils d'une bonne insertion y compris la connaissance des usages, des principes et des valeurs de notre société ", créé à l'initiative de la DAIC $^{8}$ - Direction de l'accueil, de l'intégration et de la citoyenneté dépendante du ministère de l'Intérieur, associée à la DGLFLF ${ }^{9}$, la délégation générale à la langue française et aux langues de France (rattachée au Ministère de la Culture) - place la langue au niveau d'un outil d'intégration. Les politiques linguistique et migratoire sont ici en étroite relation, ce qui confère à ce sigle relativement récent (2011) une coloration politique et polémique ${ }^{10}$.

Les adultes en formation linguistique sont signataires d'un Contrat d'Accueil et d'Intégration ${ }^{11}$. En signant ce contrat, l'adulte migrant s'engage à suivre une formation civique et une formation linguistique prescrite en fonction des résultats obtenus lors de tests réalisés par des

8. Devenue depuis 2013 la DAAEN (direction de l'accueil, de l'accompagnement des étrangers et de la nationalité). Pour plus d'informations : http://www. linguistiquefrançaise.org (site consulté le 20 novembre 2016).

9. Voir à ce propos : http://www.culturecommunication.gouv.fr/Politiquesministerielles/Langue-francaise-et-langues-de-France (site consulté le 20 novembre 2016).

10. Voir à ce propos l'article « FLIC ? Le français, langue d'une immigration contrôlée » : https://blogs.mediapart.fr/edition/les-invites-de-mediapart/article/091111/flic-lefrancais-langue-dune-integration-contro (site consulté le 20 novembre 2016).

11. Depuis le $1^{\text {er }}$ juillet 2016, le CAI est remplacé par le Contrat d'Intégration Républicaine (CIR). 
auditeurs de l'Office Français de l'Immigration et de l'Intégration ${ }^{12}$. Si le test n'est pas concluant aux yeux des critères établis par le ministère de l'Intérieur, l'adulte se voit alors prescrire une formation linguistique gratuite dans une structure labellisée FLI par l'État (il y a cinq centres labellisés en Bretagne).

Les compétences orales (compréhension, production et interaction) sont particulièrement travaillées afin d'obtenir un diplôme Initial en Langue Française (DILF) correspondant au niveau A1.1 $1^{13}$ du Cadre Européen Commun de Références pour les Langues ${ }^{14}$. Ce niveau présentant un volet oral très important répond aux particularités du public visé par ces formations et des objectifs d'intégration en France tels que définis par le Référentiel FLI.

«Son enseignement tient compte : - des "points de départ" de chacun des apprenants. Ces prérequis peuvent être très disparates, selon que ceux-ci sont ou non lecteurs et/ou scripteurs et selon leur univers linguistique originel, - de l'hétérogénéité des publics, caractéristique constituant elle-même l'une des conditions de la pédagogie ${ }^{15}$.»

"Le "Français langue d'intégration" correspond à un usage acquis par immersion : il privilégie la forme orale (...). C'est une langue destinée à devenir la langue courante des apprenants. Elle n'est pas enseignée comme une langue étrangère, mais est progressivement intériorisée. »

Les compétences orales ont ainsi une place de choix dans la formation, mais il est tout de même prudent de s'interroger sur l'idée d'une suffisance de l'immersion, d'une acquisition naturelle de la langue qui finirait par devenir courante. Pour s'intégrer, selon le ministère, il est nécessaire de parler français. Mais alors de quel français s'agit-il ? Et plus précisément si on se pose la question d'un point de vue phonolo-

12. Site officiel de l'OFII : http://www.ofii.fr/ (site consulté le 20 novembre 2016).

13. Au moment de l'enquête, il est depuis d'un niveau A1.

14. Version française du Cercl, Conseil de l'Europe, Cadre européen commun de référence pour les langues vivantes : apprendre, enseigner, évaluer, Paris, Éditions Didier, 2001.

15. Anne ViCHER (dir.), Référentiel FLI (Français Langue d'Intégration), Paris, Direction de l'Accueil, de l'Intégration et de la citoyenneté, 2011. 
gique, avec quel accent ? Existe-t-il un accent normé ? ou perçu comme normé ? Faudrait-il alors «absorber et reproduire les contours » de la langue pour reprendre une formule de Christine Deprez ${ }^{16}$ ?

Avant de clore cette série de précisions contextuelles, il semble nécessaire de mentionner que le concept de plurilinguisme, ou plus exactement sa prise en compte par les apprenants et les formateurs ${ }^{17}$, est en effet sous-jacent à l'ensemble de cette recherche. Des adultes qui parlent une ou plusieurs langues (en moyenne, les stagiaires rencontrés expliquent qu'ils parlent trois à quatre langues) ont l'injonction d'apprendre le français, obligation qui ne prend pas (ou peu) en compte les désirs ou non d'apprendre, la diversité des trajectoires professionnelles et personnelles ou les disponibilités psychologiques. Face à cette situation d'apprentissage, des mécanismes se mettent alors en place : conformité à la norme attendue et fantasmée, résistances, sentiments d'illégitimité, d'insécurité linguistique, d'acculturation, choix conscients ou non de transmission dans le cadre des politiques linguistiques familiales etc.

Entrer en formation linguistique par obligation confère des spécificités aux interactions. Ce sont ces spécificités, étudiées sous l'angle de l'oralité et de ses représentations que nous nous attachons à comprendre et à analyser.

L'étude des interactions ${ }^{18}$ se situant au cœur de cette recherche, je m'inscris dans une démarche ethnographique de l'étude de la langue, ou autrement dit dans le cadre de l'ethno-sociolinguistique définie par Philippe Blanchet ${ }^{19}$. L'objectif est en effet de saisir sur le terrain des interactions répondant de manière pertinente aux questions d'ordre sociolinguistique et d'en identifier les mécanismes.

16. Christine DePrEZ, op. cit.

17. Ma recherche de terrain s'attachant à étudier les interactions, le contexte d'apprentissage/enseignement serait incomplet s'il n'était pas question d'interroger également le volet « formateurs » via des entretiens individuels.

18. Erving GOFFMAN, La mise en scène de la vie quotidienne, tome 1 : La présentation de soi, Paris, Les Éditions de Minuit, 1973.

19. Philippe BLANCHET, Linguistique de terrain: méthode et théorie: une approche ethnosociolinguistique, Rennes, Presses Universitaires de Rennes, 2000. 


\section{L'enquête de terrain}

Les lieux d'enquête sont des centres de formation pour adultes labellisés FLI par l'État suite à un appel d'offres basé sur un cahier des charges relativement dense. Ainsi, en appliquant les critères de labellisation, les formations linguistiques proposées doivent être dispensées par des formateurs diplômés en Français langue étrangère et formés au FLI par le biais de formations internes.

Le public d'adultes allophones en contexte exolingue, est fortement marqué par l'hétérogénéité : horizons géographiques, origines sociales, culturelles, degrés de scolarisation et d'alphabétisation. Les groupes de stagiaires sont très hétérogènes : ils se composent en moyenne d'une quinzaine d'adultes de tous âges et de toutes nationalités. Bien que certaines zones d'origine soient plus représentées que d'autres (Syrie, pays du Caucase, Asie du Sud), j'ai rencontré des stagiaires de plus d'une vingtaine de nationalités. Les âges sont très variés, avec des jeunes adultes de 18 ans jusqu'à des stagiaires de plus de 65 ans. Enfin, les profils sociolinguistiques des stagiaires sont très divers, ainsi que les niveaux d'alphabétisation, de scolarisation et de compétences professionnelles en fonction des variables sociales, géographiques et culturelles.

Comment s'est déroulée l'enquête et quels choix ont été faits en matière d'outils de recueil d'informations auprès des stagiaires et des formateurs? Une approche plurielle a été mise en place, mêlant des temps d'observation participante à des outils méthodologiques tels que le questionnaire à visée exploratoire, un travail de co-construction de biographies langagières (pour un nombre limité de stagiaires) et des entretiens semi-directifs individuels auprès des stagiaires et des formatrices. Les biais tels que le paradoxe de l'observateur, les négociations inhérentes aux échanges et les différentes postures du jeune chercheur sur le terrain ont été explicités et analysés dans un travail de réflexivité et de-socio-analyse ${ }^{20}$.

20. Myriam Dupouy, « L'exercice de (re)présentation de soi lors d'une enquête de terrain : négociation ? enjeu épistémologique ? ", Social and Human Sciences Web of conferences, $n^{\circ} 20$, Proceedings ICODOC 2015. 


\section{Les transcriptions : un enjeu méthodologique et épistémo- logique}

Les transcriptions interviennent à un moment charnière du travail de thèse : l'enquête a été menée, les premières analyses ont été amorcées, mais l'analyse de contenu plus globale reste à construire en partie sur la base des transcriptions d'entretiens et d'interactions in situ. Cette étape clé, bien que marquée "méthodologie », a soulevé un nombre d'interrogations qui se sont avérées fructueuses d'un point de vue épistémologique, grâce notamment au concept de stylisation vocale.

Les interrogations de départ étaient liées premièrement au niveau de langue des stagiaires et aux possibilités de retranscrire fidèlement mais toutefois de manière compréhensible nos échanges lors des entretiens. Fallait-il transcrire exactement nos propos en y incluant les longs temps de pause, les erreurs de langue légitimes selon les niveaux de compétence ? Cette possibilité a été écartée, rendant trop complexe la lecture pour une personne extérieure à l'enquête, et donc peu compréhensible par un lecteur non averti. Ensuite, pour abonder dans ce refus de la " transcription mot à mot», elle n'aurait certainement pas été fidèle aux échanges dans lesquels le non-verbal a eu une place très importante, notamment avec les stagiaires ayant débuté depuis peu leur formation linguistique et ayant peu d'aisance en français.

Dans un second temps - et après avoir souhaité mettre à profit le travail de biographies langagières mené auprès de certains stagiaires, est apparue une question relevant du domaine de la faisabilité : comment produire des textes du type récit pour chaque stagiaire ? Cet outil qui avait été pensé au départ comme central dans le travail de terrain s'est avéré plus complexe à mettre en place dans une démarche globale (et dépassant donc la phase exploratoire) que je souhaitais vivement mettre en place qui n'a pu se concrétiser pour plusieurs raisons : la première est que je n'ai pas pu suivre les stagiaires sur une même durée, donc je n'ai pas pu mener un travail sur le long terme comme l'exige la biographie langagière ${ }^{21}$ (outil utilisé en formation pour adultes qui nécessite beaucoup d'échanges, de coopération, de prise de recul et de « navette » entre les co-constructeurs). La seconde raison provient 
d'un respect de l'éthique exigée par le chercheur: le manque de temps, l'impossibilité de faire de retours aux personnes sollicitées et donc de décider seule et arbitrairement si le récit était conforme à nos échanges et à leur souhait de mise en récit (ce qui n’a pas été réalisable en raison d'une caractéristique des formations FLI : les entrées et sorties permanentes des stagiaires pour diverses raisons familiales ou professionnelles par exemple rendent le travail sur le moyen ou long terme peu réalisable), contrairement donc à ce qui est convoqué dans la biographie langagière, à savoir la co-construction, l'accompagnement et la possibilité d'évolution du discours.

La troisième des interrogations était liée à la difficulté de la prise en compte et des transcriptions des «imitations » réalisées par les stagiaires en contexte de formation et lors des entretiens. Le terme d'« imitations » renvoie ici à la reproduction par les stagiaires de la manière de parler des autres locuteurs du groupe ou des personnes non présentes mais convoquées au discours direct (en général). Par ailleurs, n’ayant pas la possibilité de recueillir des traces audio ou vidéo des interactions en formation (hors entretiens) mais seulement celle de prendre des notes à la façon d'un ethnographe, j’ai relevé les limites qu'auraient l'exploitation et l'analyse par la perte d'une grande part de la richesse des interactions en passant « mécaniquement » par l'écrit, sans pouvoir rendre « audible, visible » ce qui se jouait réellement lors de ces moments d'imitation.

Donc, comme le rapport à la langue est au cœur de mes recherches, mais que c'est également le code complexe, la forme que je recueille, je me suis mise à chercher dans diverses directions afin de trouver des concepts et outils me permettant de «transcrire » selon mes critères et problématiques les entretiens et les observations d'ordre épilinguistique.

\section{La stylisation vocale}

Dans un article intitulé «Stylisation vocale et autres procédés dialogiques dans la socialisation langagière adolescente » paru en $2007^{22}$,

22. Cyril Trimaille, «Stylisation vocale et autres procédés dialogiques dans la socialisation langagière adolescente ", Cabiers de praxématique, n 49, 2007, p. 183-206. Pour une vue plus complète de ses travaux : http://lidilem.u-grenoble3.fr/membres/ Consultation-d-une-fiche-membre?mem_id=40 (site consulté le 20 novembre 2016). 
Cyril Trimaille propose d'analyser les procédés dialogiques mobilisés par les jeunes dans un contexte donné ainsi que les processus d'identification qui en découlent. Son corpus est constitué d'enregistrements de discussions entre jeunes en sa présence et sans lui.

En s'appuyant et en reprenant le concept d'ethos (défini par Barthes comme constitué de « tous les traits de caractère que l'orateur doit montrer pour faire bonne figure $\left.{ }^{23} »\right)$, il procède à une analyse de discours fine englobant aussi bien les aspects syntaxique, phonologique que lexical. Il y ajoute une dimension qui nous intéresse tout particulièrement qui relève du voco-prosodique.

Par stylisation vocale, on comprend une reprise de structure mémorisée, qu'elle soit immédiate ou différée, sur un mode parodique (stéréotypes par exemple d'un accent perçu comme précieux) ou non. Ainsi « avec la stylisation, de la parodie du corps parlant - de l'ethos on passe à la dévaluation de l'être social » car il y a bien un «lien entre [la] parodie de la voix et [l] évaluation de l'être 'complet' ${ }^{24}$ ».

La stylisation apparait davantage comme la parodie d'une altérité, dans une perspective d'expression schématique du ressenti qu'une démarche d'analyse et d'imitation. Je vais croiser ces propositions avec mon problème à résoudre et par conséquent tenter de montrer la pertinence de leur adaptation.

Certains stagiaires, le plus souvent en contexte de groupe, reprennent les manières de parler de leur formatrice, de leurs collègues stagiaires ou encore de personnes extérieures à la formation. Les extraits choisis sont issus du corpus d'interactions en contexte classe et du corpus d'entretiens individuels (avant codage de transcription).

- Extrait 1 (interactions - contexte classe)

Deux stagiaires thailandaises qui ne se sont pas vues depuis un moment se retrouvent assises l'une à côté de l'autre en formation : elles prennent des nouvelles de leurs familles respectives en thai et passent au français à la demande de leur formatrice. À ce moment-là, deux

23. Roland BARTHES, «L'ancienne rhétorique », Communications 16, 1966, p. 212. Cité par C. Trimaille, $i b i d$.

24. C. Trimaille, ibid. 
stagiaires marocaines imitent alors des chants d'oiseaux en joignant des gestes à leurs paroles (font battre leurs mains comme des ailes et clignent des yeux rapidement). Rire général. Je demande pourquoi c'est drôle et pourquoi elles font le rapprochement avec des oiseaux. L'une d'entre elles explique que pour elle, les Asiatiques parlent tous comme des petits oiseaux, que c'est aigu, rapide et imite à nouveau le chant des oiseaux. Rire à nouveau, d'autres stagiaires approuvent cette comparaison.

\section{-Extrait 2 (entretien individuel, FAT. F, 29 ans)}

Une stagiaire marocaine exprime son souhait de parler français « $100 \%$ », « avec l'accent et tout », comme la formatrice qu'elle imite « bonjour tout le monde, comment ça va aujourd'hui ? » en exagérant le schéma intonatif et en me demandant si elle le «fait bien».

\section{- Extrait 3 (entretien individuel, PET. F, 31 ans)}

Une stagiaire tchétchène qui explique qu'elle aimerait parler au téléphone sans que ça se «voie ». Elle explique aussi qu'à l'école de son fils ça serait mieux de "parler avec l'accent», et elle imite une vraie maman française « mon chéri, tu as mangé qui [pour quoi] le midi ? » en exagérant également la montée mélodique et en se penchant comme si elle parlait réellement à un jeune enfant.

Dans ces trois cas, les imitations semblent répondre à des schémas bien distincts. Dans le premier extrait, il pourrait s'agir d'une stylisation parodique, c'est-à-dire nourrie par l'envie de reproduire un stéréotype selon lequel les apprenants asiatiques seraient plus durs à comprendre que les autres stagiaires (difficultés de prononciation de certains phonèmes et prégnance des tons de la L1 par exemple), ou bien encore une tentative de différenciation entre les quatre femmes. Le rire provoqué par ces « imitations » n'est pas non plus anodin : visiblement, les autres personnes présentes dans la salle participent à la «blague » et à ses effets en donnant l'impression d'y adhérer. 
Dans le second extrait, le contexte est différent, il s'agit d'une stagiaire qui n'a jamais été scolarisée dans son pays d'origine, mariée à un Français qui l'aide à étudier afin qu'elle apprenne à lire, à écrire et à parler en français. Sa pratique de l'oral est bonne, elle a visiblement mis beaucoup de stratégies en place afin de rendre invisibles ses grandes difficultés face au monde de l'écriture et de la lecture. Parler français est une nécessité afin de s'intégrer dans sa belle-famille, une manière de se préparer à un futur emploi, exprime-t-elle en entretien individuel. Mais «bien parler» selon ses critères, c'est parler 100\% avec l'accent, ce qui revient par exemple à parler « comme » sa formatrice, française d'origine. Ici, le français, ou plutôt sa norme, est mythifié, il n'y a pas plus d'explications, mais l'utilisation du terme «100\%» exclut de fait une quelconque place pour la langue d'origine. Bien parler français serait donc synonyme d'effacer les traces (que l'on retrouve dans l'accent notamment) de son origine. Cet entretien, très long et très riche, a déjà révélé - via une première analyse de contenu, la prégnance d'un fort sentiment d'insécurité linguistique, renvoyant le locuteur en situation d'apprentissage à une mauvaise image de sa première langue, la darija, non conforme à la "vraie» langue, l'arabe classique qui est langue officielle au Maroc. La stagiaire se représente la langue française comme ayant un type d'accent (lequel ?), non pourvue de variations et ayant pour modèle la langue parlée par la formatrice. La question des usages et des variétés sociolinguistiques des locuteurs du français est peu abordée en formation, faute de temps et de matériel ad hoc selon les professionnelles interrogées, ce qui vient renforcer ce statut de formateur-locuteur-modèle.

Dans le troisième extrait (la confusion entre les interrogatifs « qui » et «quoi » importe peu ici), le procédé de stylisation illustre une envie, un fantasme : parler comme cette autre maman, qu'elle nous présente comme une maman française, "native », signifierait parler comme les autres, donc par recoupement, ne pas se distinguer des autres mères à la sortie de l'école. Cette hypothèse recoupe le fait qu'elle ne souhaite pas que ce qu'elle perçoit comme étant son accent se «voie» au téléphone et révélerait une volonté de se «fondre » dans un groupe représenté comme homogène et légitime et donc de passer inaperçue.

On remarque ici une utilisation du vocabulaire lié au visuel pour décrire l'accent que je retrouve à plusieurs reprises lors de l'enquête : 
est-ce dû à un lexique restreint aux choses de la vie quotidienne, ce qui serait prévisible à ce stade d'apprentissage, ou bien est-ce révélateur d'une représentation visuelle, corporelle et donc intime des accents?

Ces trois extraits illustrent les différentes possibilités d'évaluer l'autre par un discours, mais aussi et surtout de s'évaluer soi-même en tant que locuteur porteur d'une identité plurielle et plurilingue. Il y aurait donc, in fine, une mise à distance via la stylisation vocale, surtout lorsqu'elle repose sur des stéréotypes, ces mises à distance étant elles-mêmes chargées d'idéologies politiques, sociales et culturelles. En reprenant les propos d'un autre selon ses représentations personnelles faisant elles-mêmes écho à des représentations collectives, on en extrait une composante, l'accent, pour se différencier, ou bien pour différencier un groupe de soi. Les marques d'altérité et/ou de proximité viennent alors renforcer le caractère identitaire de l'accent ou de son absence, qu'il s'agisse de production ou de réception.

Ces mises en relief via la stylisation vocale viennent confirmer des analyses plus globales ${ }^{25}$ montrant des représentations et attitudes envers les accents qui oscillent entre une volonté de s'intégrer (ici de s'assimiler ?) et donc de s'approprier une norme plutôt monophonique du français (discours de l'institution) et un sentiment de résistance, c'est-à-dire de conserver son accent en le positionnant comme un marqueur d'identité, une bannière avec laquelle on ne négocie pas.

25. Myriam DupOuY, «Représentations des accents en formation linguistique pour adultes migrants : quelle acceptation de l'hétérogénéité ? ", Réseau Francophone de Sociolinguistique (à paraître). 